\title{
Imaging optically scattering objects with ultrasound-modulated optical tomography
}

\author{
Sri-Rajasekhar Kothapalli, ${ }^{1}$ Sava Sakadžić, ${ }^{2}$ Chulhong Kim, ${ }^{1}$ and Lihong V. Wang ${ }^{1 * *}$ \\ ${ }^{1}$ Department of Biomedical Engineering, Optical Imaging Laboratory, Washington University in St. Louis, \\ Campus Box 1097, One Brookings Drive, St. Louis, Missouri 63130-4899, USA \\ ${ }^{2}$ Currently with the Athinoula A. Martinos Center for Biomedical Imaging, Massachusetts General Hospital, Harvard \\ Medical School, Charlestown, Massachusetts 02129, USA \\ *Corresponding author: lhwang@biomed.wustl.edu
}

Received April 24, 2007; revised June 27, 2007; accepted July 2, 2007; posted July 6, 2007 (Doc. ID 82399); published August 2, 2007

\begin{abstract}
We show the feasibility of imaging objects having different optical scattering coefficients relative to the surrounding scattering medium using ultrasound-modulated optical tomography (UOT). While the spatial resolution depends on ultrasound parameters, the image contrast depends on the difference in scattering coefficient between the object and the surrounding medium. Experimental measurements obtained with a CCDbased speckle contrast detection scheme are in agreement with Monte Carlo simulations and analytical calculations. This study complements previous UOT experiments that demonstrated optical absorption contrast. (C) 2007 Optical Society of America

OCIS codes: $030.6140,110.6150,110.7050,110.7170,170.3880$.
\end{abstract}

Optical absorption and scattering of visible and nearinfrared light in soft biological tissue are related to tissue biochemical composition and morphology. Thus, optical imaging modalities are capable of providing structural, functional, and molecular information about the tissue [1-4]. Variations in the scattering coefficient can provide information about neuronal activity $[2,5]$ and diseased tissue [6]. However, it remains a challenge to provide optical scattering contrast in deep tissue (beyond the ballistic regime) with high spatial resolution. Diffuse optical tomography (DOT) can quantify the spatial distribution of optical absorption and scattering coefficients in tissue but suffers from poor spatial resolution $[2,7,8]$. Photoacoustic tomography (PAT) and ultrasound-modulated optical tomography (UOT) are ultrasound-mediated imaging modalities that provide high optical contrast and high ultrasonic resolution [9]. PAT, however, is mostly sensitive to light absorption. Therefore, there is a need to explore the feasibility of UOT for imaging optical scattering contrast in deep tissue.

In UOT, a focused ultrasound wave encodes photons passing through the ultrasonic beam. The ultrasound-modulated (encoded) light is measured using a variety of detection schemes [9-14]. The intensity of ultrasound-modulated light is related to the local optical properties of the tissue where ultrasound interacts with light [15-17].

In this Letter, for the first time to our knowledge, we demonstrate the capability of UOT to image objects having various optical scattering coefficents (including nearly transparent objects) buried in an optically strong scattering background medium. We first experimentally acquire one-dimensional (1D) images of absorbing and scattering objects. We then compare the experimental data with Monte Carlo (MC) and analytical calculations. Finally, we experimentally acquire a two-dimensional (2D) image of optically scattering objects.
We use the speckle contrast detection technique in combination with intense acoustic bursts and transmission geometry to obtain UOT images with a good signal-to-noise ratio (SNR) $[18,19]$. As shown in Fig. 1 , a focused ultrasound transducer (Ultran, VHP1001-138; $1 \mathrm{MHz}$ central frequency, $25.4 \mathrm{~mm}$ lens diameter, $38 \mathrm{~mm}$ focal length, $2 \mathrm{~mm}$ focal zone width, and $20 \mathrm{~mm}$ focal zone length) insonifies the tissue phantom (scattering medium) through water along the $Z$ axis (ultrasonic axis). The laser (Coherent, Verdi; $532 \mathrm{~nm}$ ) delivers light to the sample during the propagation of ultrasonic burst in the medium with an average intensity of $12 \mathrm{~mW} / \mathrm{cm}^{2}$, which is within the American National Standards Institute (ANSI) safety limits [9]. The optical axis is chosen as the $X$ axis, whose origin coincides with the point of incidence of the laser beam on the scattering slab. A function generator (Agilent, 33250A) synthesizes $2 \mathrm{~ms}$ bursts of $1 \mathrm{MHz}$ sinusoidal signals that are subsequently amplified by an rf amplifier (ENI, Inc., 325LA) to drive the ultrasound transducer. The ultrasound peak pressure measured with a needle hydrophone at the focus is $1.5 \mathrm{MPa}$. This pressure translates into a mechanical index of 1.5 and is within the safety limits [9]. The burst initiation pulse triggers a pulse-delay generator (Stanford Research, DG535) to produce two CCD (Basler, A312f; 12-bit, $640 \times 480$ pixels) trigger pulses per second, separated

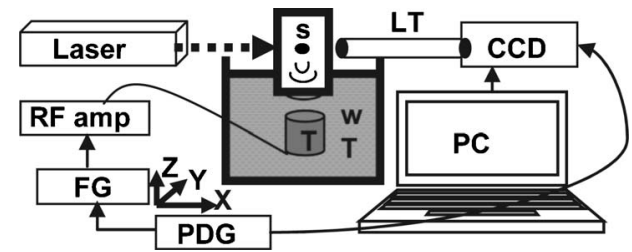

Fig. 1. Experimental setup: CCD, CCD camera; RF amp, $\mathrm{RF}$ amplifier; FG, function generator; $\mathrm{PDG}$, pulse delay generator; T, ultrasound transducer; WT, water tank; S, sample; LT, lens tube; PC, personal computer. 
by $500 \mathrm{~ms}$. In response to the first trigger pulse, the CCD camera captures one ultrasound burstsynchronized laser speckle image. In response to the second trigger pulse, the CCD camera captures another speckle image without ultrasound burst. The exposure time of the CCD camera is set to $2 \mathrm{~ms}$. The speckle contrast change (SCC) between these two speckle images is approximately proportional to the intensity of the ultrasound-modulated light $[12,18]$. At each position of the ultrasonic transducer, six measurements of SCC are averaged. By scanning the ultrasonic beam across the scattering medium, we form an SCC image of optical inhomogeneities inside the medium at ultrasonic resolution.

The background tissue phantoms and buried objects are made from gelatin, water, and Intralipid (20\% Liposyn II, Intravenous Fat Emulsion Hospira, Inc.). The tissue phantom is an optically scattering slab $10 \mathrm{~cm}$ wide in the $Y$ (ultrasound scanning axis) and $Z$ directions and $2 \mathrm{~cm}$ thick in the $X$ direction. The optical reduced scattering and absorption coefficients of the scattering slab (background medium) are $\mu_{s}^{\prime}=10 \mathrm{~cm}^{-1}$ and $\mu_{a}=0.1 \mathrm{~cm}^{-1}$, respectively. Five different optical scattering objects are prepared with approximate dimensions of 2,2 , and $20 \mathrm{~mm}$ in the $X$, $Y$, and $Z$ directions, respectively. The reduced scattering coefficients $\mu_{s}^{\prime}$ of the five objects are $30,18,10,5$, and $0.5 \mathrm{~cm}^{-1}$ (nearly transparent object). The optical absorption coefficient of all scattering objects is the same as that of the background medium ( $\mu_{a}$ $\left.=0.1 \mathrm{~cm}^{-1}\right)$. By adding Trypan Blue dye to the Intralipid-gelatin solution, we prepare highly absorbing objects $\left(\mu_{a}=100 \mathrm{~cm}^{-1}\right)$ with the same dimensions as the scattering objects. The optical properties are measured by an oblique incidence reflectometer at $532 \mathrm{~nm}$ wavelength [20]. Several tissue phantoms are prepared by embedding different scattering objects and absorbing objects inside the scattering slab at a depth of $X=1 \mathrm{~cm}$.

Figure 2(a) shows two experimental 1D images [one SCC image and one total optical intensity (DC) image that is averaged over all CCD pixels during the time when ultrasound is off] of one absorbing object and one nearly transparent object separated by $22 \mathrm{~mm}$ along the $Y$ axis. The local decrease in SCC (45\%) when the ultrasound is focused on the absorbing object represents the decrease in detected modu-
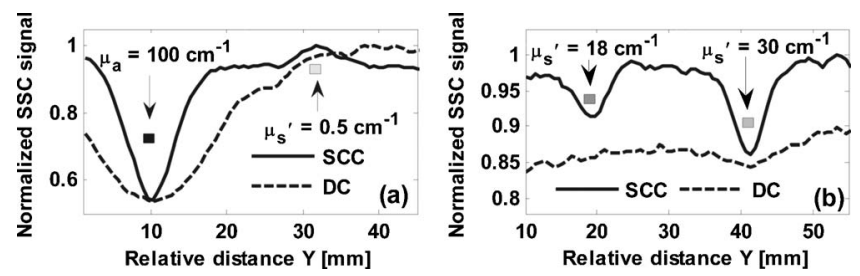

Fig. 2. (a) Normalized 1D SCC image and total optical intensity (DC) image of one absorbing object $(y=10 \mathrm{~mm})$ and one nearly transparent object $(y=32 \mathrm{~mm})$ separated by $22 \mathrm{~mm}$. Positions corresponding to both objects are marked with squares. (b) Normalized 1D SCC image and DC image of two scattering objects $(y=19 \mathrm{~mm}$ and $y=41 \mathrm{~mm})$ separated by $22 \mathrm{~mm}$. Positions corresponding to both objects are marked with squares. lated optical intensity due to strong absorption of light by the object. In contrast, when ultrasound is focused on the transparent object, the SCC increases significantly $(5 \%)$. Since the transparent object and the background have the same $\mu_{a}$, this increase in SCC can be attributed to the lower $\mu_{s}^{\prime}$ of the transparent object than that of the background. Figure 2(b) shows an experimental 1D SCC image and a DC image of two scattering objects, whose reduced scattering coefficients $\left(\mu_{s}^{\prime}=18 \mathrm{~cm}^{-1}\right.$ and $\left.\mu_{s}^{\prime}=30 \mathrm{~cm}^{-1}\right)$ are greater than that of the background $\left(\mu_{s}^{\prime}=10 \mathrm{~cm}^{-1}\right)$. The two objects are separated by $22 \mathrm{~mm}$ along the $Y$ axis. The SCC decreases by $\sim 8 \%$ and $\sim 15 \%$, respectively, when the ultrasound is focused on each scattering object. In both Figs. 2(a) and 2(b), the SCC images are more sensitive to optical heterogeneity than the DC images and provide better spatial resolution and higher contrast.

Figure 3 compares the image contrasts of five optically scattering objects-with $\mu_{s}^{\prime}$ of $30,18,10,5$, and $0.5 \mathrm{~cm}^{-1}$, respectively-obtained from the experiments and MC simulations, as a function of $\mu_{s}^{\prime}$. The MC simulations and the experiments share the same physical parameters for the ultrasound, the light source, the background medium, and the buried objects. The MC algorithm can model the power spectral density of ultrasound-modulated multiply scattered light when a focused ultrasound field is present in a heterogeneous optically scattering medium [21]. Using the MC algorithm, we obtain the modulation depth $\left[M\left(\mu_{s}^{\prime}\right)\right]$ at each detector position for the five different optically scattering objects, where $M\left(\mu_{s}^{\prime}\right)$ is the ratio of the modulated to the unmodulated light intensities. In all simulations, the focus of the ultrasound is placed at the center of the scattering object. The relative change in modulation depth for an object with $\mu_{s}^{\prime},\left[M\left(\mu_{s}^{\prime}\right) / M\left(10 \mathrm{~cm}^{-1}\right)\right]-1$, is modeled as the image contrast, where $10 \mathrm{~cm}^{-1}$ is the $\mu_{s}^{\prime}$ of the background medium. The image contrast is positive for scattering objects with $\mu_{s}^{\prime}<10 \mathrm{~cm}^{-1}$ and negative for scattering objects with $\mu_{s}^{\prime}>10 \mathrm{~cm}^{-1}$; it decreases with increasing $\mu_{s}^{\prime}$ of the buried object. The experimental measurements and the MC results are in good agreement, although the ultrasound pressure is beyond the limit required by the MC model.

The trend observed in Fig. 3 can be explained analytically. For moderate ultrasound pressures and in the weak scattering approximation, we have SCC $\approx M \approx(1 / 2)\left(C_{n, \bar{s}}+C_{d, \bar{s}}+C_{n d, \bar{s}}\right)$, where $C_{n, \bar{s}}, C_{d, \bar{s}}$, and $C_{n d, \bar{s}}$ represent contributions due to (1) modulated op-

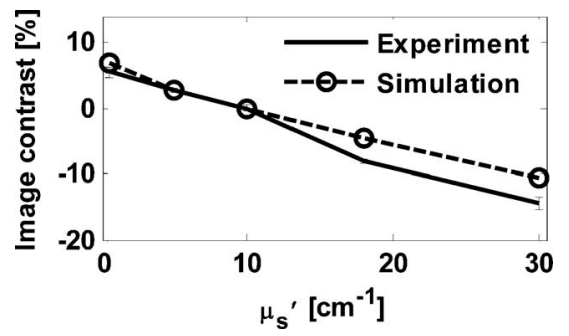

Fig. 3. Image contrast of different optically scattering objects as a function of $\mu_{s}^{\prime}$. 
tical index of refraction, (2) modulated displacement of optical scatterers, and (3) anticorrelation between the two mechanisms of modulation ( $C_{n d, \bar{s}}$ is negative); $\bar{s}$ represents the average path length $s$ within the ultrasound field $[12,17,18]$. As $\mu_{s}^{\prime}$ increases while $\bar{s}$ is held approximately constant at $2.5 \mathrm{~mm}$ (focal beam width), $C_{d, \bar{s}}$ and $-C_{n d, \bar{s}}$ increase approximately linearly with the number of scattering events, and $C_{n, \bar{s}}$ initially decreases due to smaller interaction lengths (optical free paths) and then increases due to strong correlations between ultrasound induced optical phase increments along different free paths. However, the change of $-C_{n d, \bar{s}}$ dominates that of $C_{n, \bar{s}}$ $+C_{d, \bar{s}}$. As a result, the sum $C_{n, \bar{s}}+C_{d, \bar{s}}+C_{n d, \bar{s}}$ and hence SCC decreases.

Figure 4(a) shows 2D imaging of two optically scattering objects that have $\mu_{s}^{\prime}=30 \mathrm{~cm}^{-1}$ and approximate dimensions of 2,2 , and $20 \mathrm{~mm}$ in the $X, Y$, and $Z$ directions, respectively. The objects are separated by $10 \mathrm{~mm}$ along the $Y$ axis and embedded in the $x$ $=1 \mathrm{~cm}$ plane inside the $2 \mathrm{~cm}$ thick scattering slab. Figure 4(b) presents a 2D image based on the normalized SCC in shades of gray. A negative SCC of $15 \%$ is measured at the centers of both objects. The results from both Figs. 2 and 4 show that the lateral spatial resolution, defined as the one-way distance between the $25 \%$ and $75 \%$ points of the SCC, is $\sim 2 \mathrm{~mm}$ for all scattering and absorbing objects, which is comparable with the ultrasonic beam waist.

In summary, this study demonstrates the potential of UOT to image both optical scattering and absorption properties of soft biological tissue. Our results show that the image contrast depends on the reduced scattering coefficients of optically scattering objects,

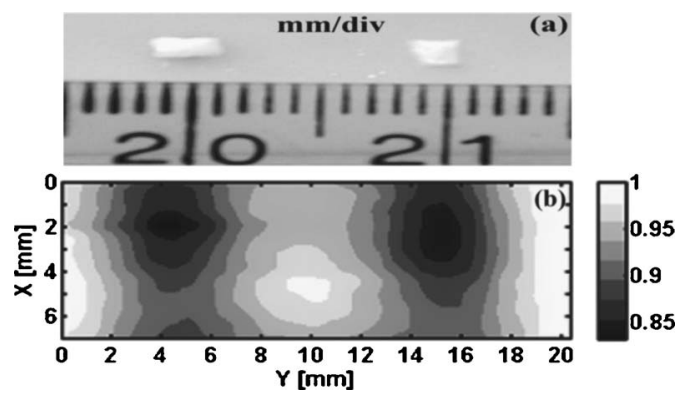

Fig. 4. (a) Photograph of two scattering objects having $\mu_{s}^{\prime}=30 \mathrm{~cm}^{-1}$. (b) Corresponding $2 \mathrm{D}$ image based on normalized SCC. whereas the resolution is determined by the ultrasound parameters. Our results also show that ultrasound-modulated light is more sensitive to scattering objects than nonmodulated transmitted light.

We thank A. Garcia-Uribe for measuring optical properties of the tissue phantoms. This research is supported by the National Institute of Health grants R33 CA 094267 and R01 CA106728.

\section{References}

1. H. F. Zhang, K. Maslov, G. Stoica, and L.-H. Wang, Nat. Biotechnol. 24, 848 (2006).

2. D. A. Boas, D. H. Brooks, E. L. Miller, C. A. DiMarzio, M. Kilmer, R. J. Gaudette, and Q. Zhang, IEEE Signal Process. Lett. 18, 57 (2001).

3. J. G. Fujimoto, Nat. Biotechnol. 21, 1361 (2003).

4. H. Inoue, S. Kudo, and A. Shiokawa, Nat. Clin. Pract. Gastroenterol. Hepatol. 2, 31 (2005).

5. A. D. Aguirre, Y. Chen, J. G. Fujimoto, L. Ruvinskaya, A. Devor, and D. A. Boas, Opt. Lett. 31, 3459 (2006).

6. S. Srinivasan, B. W. Pogue, S. Jiang, H. Dehghani, C. Kogel, S. Soho, J. J. Gibson, T. D. Tosteson, S. P. Poplack, and K. D. Paulsen, Proc. Natl. Acad. Sci. U.S.A. 100, 12349 (2003).

7. G. Gulsen, O. Birgul, M. B. Unlu, R. Shafiiha, and O. Nalcioglu, Technol. Cancer Res. Treat. 5, 351 (2006).

8. H. Zhao, X. Gu, and H. Jiang, Opt. Commun. 238, 51 (2004)

9. L.-H. Wang, J. Dis. Mark. 19, 123 (2004).

10. S. Leveque, A. C. Boccara, M. Lebec, and H. SaintJalmes, Opt. Lett. 24, 181 (1999).

11. A. Lev and B. G. Sfez, Opt. Lett. 28, 1549 (2003).

12. J. Li, G. Ku, and L.-H. V. Wang, Appl. Opt. 41, 6030 (2002).

13. S. Sakadžić and L.-H. V. Wang, Opt. Lett. 29, 2770 (2004).

14. T. W. Murray, L. Sui, G. Maguluri, R. A. Roy, A. Nieva, F. Blonigen, and C. A. DiMarzio, Opt. Lett. 29, 2509 (2004).

15. L.-H. V. Wang, Phys. Rev. Lett. 87, 043903 (2001).

16. S. Sakadžić and L.-H. V. Wang, Phys. Rev. Lett. 96, 163902 (2006).

17. S. Sakadžić and L.-H. V. Wang, Phys. Rev. E 72, 036620 (2005).

18. R. Zemp, S. Sakadžić, and L.-H. V. Wang, Phys. Rev. E 73, 061920 (2006).

19. C. Kim, R. J. Zemp, and L-H. V. Wang, Opt. Lett. 31, 2423 (2006).

20. G. Marquez and L.-H. V. Wang, Opt. Express 1, 454 (1997).

21. S. Sakadžić and L.-H. V. Wang, Phys. Rev. E 74, 036618 (2006). 